\title{
On the variety generated by completions of representable relation algebras
}

\author{
Ian Hodkinson
}

\begin{abstract}
Maddux recently defined the variety $V$ generated by the completions of representable relation algebras. In this note, we observe that $V$ is canonical, answering Maddux's problem 1.1(3), and show that the variety of representable relation algebras is not finitely axiomatisable over $V$.
\end{abstract}

Mathematics Subject Classification. 03G15, 03C05, 05C15, 06B23.

Keywords. Non-finitely axiomatisable variety, Canonical variety, Graph, Chromatic number, Cycle.

\section{Introduction}

In a recent paper $[9,1.10]$, Maddux defined the variety $V=\mathbf{H S P}_{\text {RRA }}{ }^{c}$, where RRA is the variety of representable relation algebras and $\operatorname{RRA}^{c}=\left\{\mathfrak{A}^{c}: \mathfrak{A} \in\right.$ RRA $\}$. Here, $\mathfrak{A}^{c}$ denotes the completion of the relation algebra $\mathfrak{A}$. For details of these notions and further ones used below, see the very attractive introduction to $[9]$.

In $[9$, problem 1.1(3)], Maddux asked whether $V$ is closed under canonical extensions.

Theorem 1.1. $V$ and $\mathrm{SRRA}^{c}$ are closed under canonical extensions.

Proof. By [4, theorem 3.8], if $\mathrm{K}$ is a class of relation algebras that is closed under ultraproducts, and $\mathrm{K}^{c}=\left\{\mathfrak{A}^{c}: \mathfrak{A} \in \mathrm{K}\right\}$, then $\mathbf{S} \mathbf{K}^{c}$ and $\mathbf{H S P} \mathbf{K}^{c}$ are both closed under canonical extensions. Theorem 1.1 follows by taking $K=$ RRA, which is a variety and so closed under ultraproducts.

RA denotes the class of all relation algebras. Using the known facts that RA is closed under completions [10] but RRA is not [7], Maddux noted that

$$
\mathrm{RRA} \subsetneq V \subseteq \mathrm{RA},
$$


and he showed that $V$ contains a number of non-representable 'Monk algebras', so that the gap between RRA and $V$ is substantial. In [9, problem 1.1(1)], he asked whether $V=\mathrm{RA}$. This was answered negatively by Andréka and Németi [1], where it is shown that in fact there are continuum-many varieties lying between $V$ and RA. That might suggest that $V$ is 'nearer' to RRA than to RA, but as 'evidence' in the other direction, we show below that RRA is not finitely axiomatisable over $V$.

\section{RRA is not finitely axiomatisable over $V$}

It suffices to show that RRA contains an ultraproduct of algebras in $V \backslash$ RRA. To this end, we use a construction from [6] of relation algebras from graphs.

\subsection{Graphs}

Graphs here are undirected and loop-free. Let $G$ be a graph whose set of nodes is $N$, say. Recall that a cycle of length $l \geq 3$ in $G$ is a subset $\left\{v_{0}, \ldots, v_{l-1}\right\} \subseteq N$ of size $l$ such that $\left(v_{i}, v_{(i+1) \bmod l}\right)$ is an edge of $G$ for each $i<l$. A subset $X \subseteq N$ is said to be independent if no pair of nodes in $X$ is an edge of $G$. The chromatic number $\chi(G)$ of $G$ is the least natural number $n$ such that $N$ is the union of $n$ (possibly empty) independent sets, and $\infty$ if there is no such $n$. It is well known (see, e.g., $[2,1.6 .1])$ that $\chi(G) \leq 2$ iff $G$ has no cycles of odd length. We let + and $\sum$ denote disjoint union of graphs. Then if $G_{i}(i \in I)$ are graphs, $\chi\left(\sum_{i \in I} G_{i}\right)$ is the least upper bound (possibly $\infty$ ) of $\left\{\chi\left(G_{i}\right): i \in I\right\}$.

Let $n$ be a positive integer. Let $K_{n}$ be a complete graph with precisely $n$ nodes. Clearly, $\chi\left(K_{n}\right)=n$. Let $E_{n}$ be a graph with $\chi\left(E_{n}\right) \geq n$ and with no cycles of length at most $n$-finite examples were constructed by Erdős [3].

We use these graphs to construct some infinite graphs $\left(G_{n}^{k}, G^{k}, G^{\omega}\right)$, and compute their chromatic numbers. First define

$$
\begin{aligned}
G_{n}^{0} & =\sum_{m \geq n} E_{m}, \\
G_{n}^{k} & =G_{n}^{0}+K_{k} \quad \text { if } 0<k<\omega .
\end{aligned}
$$

Since $\left\{\chi\left(E_{m}\right): m \geq n\right\}$ is unbounded, each $G_{n}^{k}$ has chromatic number $\infty$.

Next, fix a non-principal ultrafilter $D$ over $\omega \backslash 1$. Define the ultraproduct

$$
G^{k}=\prod_{D}\left\{G_{n}^{k}: 0<n<\omega\right\}, \quad \text { for each } k<\omega .
$$

First consider the case when $k=0$. Observe that $G_{n}^{0}$ in $(2.1)$ has no cycles of length $\leq n$. Now for each $l \geq 3$, the property of having no cycles of length $l$ can be expressed by a first-order sentence, and is true for all but finitely many $G_{n}^{0}$. So by Loś's theorem, $G^{0}$ in (2.3) has no cycles of length $l$. This holds for each $l$, so in fact $G^{0}$ has no cycles at all, and hence $\chi\left(G^{0}\right) \leq 2$.

What about $\chi\left(G^{k}\right)$ for $k>0$ ? By standard ultraproduct considerations,

$$
G^{k} \cong G^{0}+K_{k}
$$

so we further obtain $\chi\left(G^{k}\right)=\max \left(\chi\left(G^{0}\right), \chi\left(K_{k}\right)\right) \leq \max (2, k)<\infty$. 
Finally let

$$
G^{\omega}=\prod_{D}\left\{G^{k}: 0<k<\omega\right\}
$$

For each $m>0, K_{m}$ embeds into $G^{k}$ for every $k \geq m$. It follows by Łoś's theorem that $K_{m}$ embeds into $G^{\omega}$, so plainly, $\chi\left(G^{\omega}\right) \geq m$. This holds for every $m$, so $\chi\left(G^{\omega}\right)=\infty$.

\subsection{Relation algebras from graphs}

Let $G$ be an infinite graph with set of nodes $N$. We write $N \times 3$ for the set $N \times\{0,1,2\}$, and $G \times 3$ for the graph whose set of nodes is $N \times 3$ and where $((v, i),(w, j))$ is an edge of $G \times 3$ iff $i \neq j$ or $(v, w)$ is an edge of $G$. In simple words, $G \times 3$ consists of three disjoint copies of $G$, with all possible edges added between the copies.

We now define a relation algebra atom structure $\alpha(G)=\left(A, C,{ }^{\smile}, I\right)$ isomorphic to one in [6, section 4] and [5, chapter 14]. We stipulate that $A=(N \times 3) \cup\left\{1^{\prime}\right\}, I=\left\{1^{\prime}\right\}, \breve{x}=x$ for every $x \in A$, and for each $x, y, z \in A$, $C(x, y, z)$ holds iff

(1) one of $x, y, z$ is $1^{\prime}$ and the other two are equal, or

(2) $\{x, y, z\} \subseteq N \times 3$ and $\{x, y, z\}$ is not independent (in the graph $G \times 3$ ).

One can check that for any graphs $G_{n}(0<n<\omega)$,

$$
\alpha\left(\prod_{D} G_{n}\right) \cong \prod_{D} \alpha\left(G_{n}\right) .
$$

We write $\mathfrak{A}(G)$ for the complex algebra of $\alpha(G)$ (see $[9,1.13]$ ). By $[8$, lemma 6.2], $\mathfrak{A}(G)$ is a relation algebra; of course it is atomic and its atom structure is $\alpha(G)$. We will need the following fact about it, from [6, theorems 10-11] or $[5$, theorems $14.12-13]$ or $($ for $\Leftarrow)[5$, exercise $14.2(7)]$. It uses our assumption that $G$ is infinite.

Fact 2.1. $\mathfrak{A}(G) \in \mathrm{RRA}$ if and only if $\chi(G)=\infty$.

\subsection{Why RRA is not finitely axiomatisable over $\boldsymbol{V}$}

Let $k<\omega$. For $0<n<\omega$, we saw that $\chi\left(G_{n}^{k}\right)=\infty$, so $\mathfrak{A}\left(G_{n}^{k}\right) \in \operatorname{RRA}$ by fact 2.1. Define the ultraproduct

$$
\mathfrak{A}^{k}=\prod_{D}\left\{\mathfrak{A}\left(G_{n}^{k}\right): 0<n<\omega\right\} .
$$

Then $\mathfrak{A}^{k} \in \mathrm{RRA}$, since RRA is a variety and closed under ultraproducts. So by definition of $V$,

$$
\mathfrak{C}^{k}=\left(\mathfrak{A}^{k}\right)^{c} \in V \text {. }
$$

By Łoś's theorem, $\mathfrak{A}^{k}$ is atomic. So (e.g., [5, remark 2.67]) its completion $\mathfrak{C}^{k}$ is isomorphic to the complex algebra of the atom structure of $\mathfrak{A}^{k}$. This atom structure is $\prod_{D} \alpha\left(G_{n}^{k}\right) \cong \alpha\left(\prod_{D} G_{n}^{k}\right)=\alpha\left(G^{k}\right)$ by $(2.6,2.5,2.3)$. Hence, $\mathfrak{C}^{k} \cong \mathfrak{A}\left(G^{k}\right)$. We saw that $\chi\left(G^{k}\right)<\infty$, so by fact $2.1, \mathfrak{C}^{k} \notin$ RRA. 
Finally let $\mathfrak{C}$ be the ultraproduct $\prod_{D}\left\{\mathfrak{C}^{k}: 0<k<\omega\right\}$. As before, this is an atomic relation algebra with atom structure isomorphic to $\prod_{D} \alpha\left(G^{k}\right) \cong$ $\alpha\left(\prod_{D} G^{k}\right)=\alpha\left(G^{\omega}\right)$, so its completion $\mathfrak{C}^{c}$ is isomorphic to $\mathfrak{A}\left(G^{\omega}\right)$. But $\chi\left(G^{\omega}\right)=$ $\infty$, so $\mathfrak{A}\left(G^{\omega}\right) \in$ RRA by fact 2.1. Then $\mathfrak{C} \subseteq \mathfrak{C}^{c} \in$ RRA, and as RRA is closed under subalgebras, we obtain $\mathfrak{C} \in$ RRA.

We can now prove our main theorem.

Theorem 2.2. RRA is not finitely axiomatisable over $V$.

Proof. We have shown that $\mathfrak{C}^{k} \in V \backslash$ RRA for $k>0$, and $\mathfrak{C}=\prod_{D} \mathfrak{C}^{k} \in$ RRA. It follows by Łoś's theorem that RRA is not finitely axiomatisable over $V$.

\section{Acknowledgements}

I thank Robin Hirsch and the anonymous referee for helpful comments.

Open Access. This article is licensed under a Creative Commons Attribution 4.0 International License, which permits use, sharing, adaptation, distribution and reproduction in any medium or format, as long as you give appropriate credit to the original author(s) and the source, provide a link to the Creative Commons licence, and indicate if changes were made. The images or other third party material in this article are included in the article's Creative Commons licence, unless indicated otherwise in a credit line to the material. If material is not included in the article's Creative Commons licence and your intended use is not permitted by statutory regulation or exceeds the permitted use, you will need to obtain permission directly from the copyright holder. To view a copy of this licence, visit http://creativecommons. org/licenses/by/4.0/.

Publisher's Note Springer Nature remains neutral with regard to jurisdictional claims in published maps and institutional affiliations.

\section{References}

[1] Andréka, H., Németi, I.: Varieties generated by completions. Algebra Universalis 80, 30 (2019)

[2] Diestel, R.: Graph theory, Graduate Texts in Mathematics, vol. 173. Springer, Berlin (1997)

[3] Erdős, P.: Graph theory and probability. Canad. J. Math. 11, 34-38 (1959)

[4] Gehrke, M., Harding, J., Venema, Y.: MacNeille completions and canonical extensions. Trans. Amer. Math. Soc. 358, 573-590 (2006)

[5] Hirsch, R., Hodkinson, I.: Relation algebras by games, Studies in Logic and the Foundations of Mathematics, vol. 147. North-Holland, Amsterdam (2002)

[6] Hirsch, R., Hodkinson, I.: Strongly representable atom structures of relation algebras. Proc. Amer. Math. Soc. 130, 1819-1831 (2002) 
[7] Hodkinson, I.: Atom structures of cylindric algebras and relation algebras. Ann. Pure Appl. Logic 89, 117-148 (1997)

[8] Hodkinson, I., Venema, Y.: Canonical varieties with no canonical axiomatisation. Trans. Amer. Math. Soc. 357, 4579-4605 (2005)

[9] Maddux, R.D.: Subcompletions of representable relation algebras. Algebra Universalis 79, 20 (2018)

[10] Monk, J.D.: Completions of boolean algebras with operators. Mathematische Nachrichten 46, 47-55 (1970)

Ian Hodkinson

Department of Computing

Imperial College London

London SW7 2AZ

UK

e-mail: i.hodkinson@imperial.ac.uk

URL: http://www.doc.ic.ac.uk/ imh/

Received: 8 July 2019.

Accepted: 7 January 2020. 